\title{
On Adjoint and Brain Functors
}

\author{
David Ellerman \\ Philosophy Department \\ University of California at Riverside
}

\begin{abstract}
There is some consensus among orthodox category theorists that the concept of adjoint functors is the most important concept contributed to mathematics by category theory. We give a heterodox treatment of adjoints using heteromorphisms (object-to-object morphisms between objects of different categories) that parses an adjunction into two separate parts (left and right representations of heteromorphisms). Then these separate parts can be recombined in a new way to define a cognate concept, the brain functor, to abstractly model the functions of perception and action of a brain. The treatment uses relatively simple category theory and is focused on the interpretation and application of the mathematical concepts. The Mathematical Appendix is of general interest to category theorists as it is a defense of the use of heteromorphisms as a natural and necessary part of category theory.
\end{abstract}

\section{Contents}

1 Category theory in the life and cognitive sciences 2

2 The ubiquity and importance of adjoints 2

3 Adjoints and universals 3

4 The Hom-set definition of an adjunction 4

5 Heteromorphisms and adjunctions 6

6 Brain functors 8

7 A mathematical example of a brain functor 12

8 Conclusion 13

9 Mathematical Appendix: Are hets really necessary in category theory? 13

9.1 Chimeras in the wilds of mathematical practice . . . . . . . . . . . . . 14

9.2 Hets as "homs" in a collage category . . . . . . . . . . . . . . . . 15

9.3 What about the homs-only UMPs in adjunctions? . . . . . . . . . . . . . . 16

9.4 Are all UMPs part of adjunctions? . . . . . . . . . . . . . . . . . . 16 


\section{Category theory in the life and cognitive sciences}

There is already a considerable but widely varying literature on the application of category theory to the life and cognitive sciences-such as the work of Robert Rosen ([31], 32]) and his followers 1 as well as Andrée Ehresmann and Jean-Paul Vanbremeersch [4] and their commentators 2

The approach taken here is based on a specific use of the characteristic concepts of category theory, namely universal mapping properties. One such approach in the literature is that of François Magnan and Gonzalo Reyes which emphasizes that "Category theory provides means to circumscribe and study what is universal in mathematics and other scientific disciplines." [26, p. 57]. Their intended field of application is cognitive science.

We may even suggest that universals of the mind may be expressed by means of universal properties in the theory of categories and much of the work done up to now in this area seems to bear out this suggestion....

By discussing the process of counting in some detail, we give evidence that this universal ability of the human mind may be conveniently conceptualized in terms of this theory of universals which is category theory. [26, p. 59]

Another current approach that emphasizes universal mapping properties ("universal constructions") is that of S. Phillips, W. H. Wilson, and G. S. Halford ([10], [30, [29]).

In addition to the focus on universals, the approach here is distinctive in the use of heteromorphismswhich are object-to-object morphisms between objects if different categories-in contrast to the usual homomorphisms or homs between objects in the same category. By explicitly adding heteromorphisms to the usual homs-only presentation of category theory, this approach can directly represent interactions between the objects of different categories (intuitively, between an organism and the environment). But it is still early days, and many approaches need to be tried to find out "where theory lives."

\section{The ubiquity and importance of adjoints}

Before developing the concept of a brain functor, we need to consider the related concept of a pair of adjoint functors, an adjunction. The developers of category theory, Saunders Mac Lane and Samuel Eilenberg, famously said that categories were defined in order to define functors, and functors were defined in order to define natural transformations [5. A few years later, the concept of universal constructions or universal mapping properties was isolated (23] and 34]). Adjoints were defined a decade later by Daniel Kan [15] and the realization of their ubiquity ("Adjoint functors arise everywhere" [24, p. v]) and their foundational importance has steadily increased over time (Lawvere [18 and Lambek [17). Now it would perhaps not be too much of an exaggeration to see categories, functors, and natural transformations as the prelude to defining adjoint functors. As Steven Awodey put it:

The notion of adjoint functor applies everything that we have learned up to now to unify and subsume all the different universal mapping properties that we have encountered, from free groups to limits to exponentials. But more importantly, it also captures an important mathematical phenomenon that is invisible without the lens of category theory. Indeed, I will make the admittedly provocative claim that adjointness is a concept of fundamental logical and mathematical importance that is not captured elsewhere in mathematics. [1, p. 179]

\footnotetext{
${ }^{1}$ See $[38,[20$, and $[21$ and their references.

${ }^{2}$ See 14 for Kainen's comments on the Ehresmann-Vanbremeersch approach, Kainen's own approach, and a broad bibliography of relevant papers.
} 
Other category theorists have given similar testimonials.

To some, including this writer, adjunction is the most important concept in category theory. [37, p. 6]

The isolation and explication of the notion of adjointness is perhaps the most profound contribution that category theory has made to the history of general mathematical ideas." [9. p. 438]

Nowadays, every user of category theory agrees that [adjunction] is the concept which justifies the fundamental position of the subject in mathematics. [36, p. 367]

\section{Adjoints and universals}

How do the ubiquitous and important adjoint functors relate to the universal constructions? Mac Lane and Birkhoff succinctly state the idea of the universals of category theory and note that adjunctions can be analyzed in terms of those universals.

The construction of a new algebraic object will often solve a specific problem in a universal way, in the sense that every other solution of the given problem is obtained from this one by a unique homomorphism. The basic idea of an adjoint functor arises from the analysis of such universals. [25, p. v]

We can use some old language from Plato's theory of universals to describe those universals of category theory (Ellerman [6]) that solve a problem in a universal or paradigmatic way so that "every other solution of the given problem is obtained from this one" in a unique way.

In Plato's Theory of Ideas or Forms $(\varepsilon \iota \delta \eta)$, a property $F$ has an entity associated with it, the universal $u_{F}$, which uniquely represents the property. An object $x$ has the property $F$, i.e., $F(x)$, if and only if (iff) the object $x$ participates in the universal $u_{F}$. Let $\mu$ (from $\mu \varepsilon \theta \varepsilon \xi \iota \varsigma$ or methexis) represent the participation relation so

$$
\text { " } x \mu u_{F} " \text { reads as " } x \text { participates in } u_{F} " .
$$

Given a relation $\mu$, an entity $u_{F}$ is said to be a universal for the property $F$ (with respect to $\mu$ ) if it satisfies the following universality condition:

$$
\text { for any } x, x \mu u_{F} \text { if and only if } F(x) \text {. }
$$

A universal representing a property should be in some sense unique. Hence there should be an equivalence relation $(\approx)$ so that universals satisfy a uniqueness condition:

$$
\text { if } u_{F} \text { and } u_{F}^{\prime} \text { are universals for the same } F \text {, then } u_{F} \approx u_{F}^{\prime} \text {. }
$$

The two criteria for a theory of universals is that it contains a binary relation $\mu$ and an equivalence relation $\approx$ so that with certain properties $F$ there are associated entities $u_{F}$ satisfying the following conditions:

(1) Universality condition: for any $x, x \mu u_{F}$ iff $F(x)$, and

(2) Uniqueness condition: if $u_{F}$ and $u_{F}^{\prime}$ are universals for the same $F$ [i.e., satisfy (1)], then $u_{F}$ $\approx u_{F}^{\prime}$.

A universal $u_{F}$ is said to be non-self-predicative if it does not participate in itself, i.e., $\neg\left(u_{F} \mu u_{F}\right)$. A universal $u_{F}$ is self-predicative if it participates in itself, i.e., $u_{F} \mu u_{F} 3$ For the sets in an iterative

\footnotetext{
${ }^{3}$ A self-predicative universal for some property is thus an impredicative definition of having that property. See 21 p. 245] where a supremum or least upper bound is referred to as giving an impredicative definition of being an upper bound of a subset of a partial order. Also Michael Makkai [27] makes a similiar remark about the universal mapping property of the natural number system.
} 
set theory (Boolos [3]), set membership is the participation relation, set equality is the equivalence relation, and those sets are never-self-predicative (since the set of instances of a property is always of higher type or rank than the instances). The universals of category theory form the "other bookend" as always-self-predicative universals. The set-theoretical paradoxes arose from trying to have one theory of universals ("Frege's Paradise") where the universals could be either self-predicative or nonself-predicative 4 instead of having two opposite "bookend" theories, one for never-self-predicative universals (set theory) and one for always always-self-predicative universals (category theory).

For the self-predicative universals of category theory (see 25] or [24 for introductions), the participation relation is the uniquely-factors-through relation. It can always be formulated in a suitable category as:

$$
\text { " } x \mu u_{F} " \text { means "there exists a unique arrow } x \Rightarrow u_{F} " .
$$

Then $x$ is said to uniquely factor through $u_{F}$, and the arrow $x \Rightarrow u_{F}$ is the unique factor or participation morphism. In the universality condition,

$$
\text { for any } x, x \mu u_{F} \text { if and only if } F(x) \text {, }
$$

the existence of the identity arrow $1_{u_{F}}: u_{F} \Rightarrow u_{F}$ is the self-participation of the self-predicative universal that corresponds with $F\left(u_{F}\right)$, the self-predication of the property to $u_{F}$. In category theory, the equivalence relation used in the uniqueness condition is the isomorphism $(\cong)$.

\section{The Hom-set definition of an adjunction}

We will later use a specific heterodox treatment of adjunctions, first developed by Pareigis [28] and later rediscovered and developed by Ellerman ([7], [8]), which shows that adjoints arise by gluing together in a certain way two universals (left and right representations). But for illustration, we start with the standard Hom-set definition of an adjunction.

The category Sets has all sets as objects and all functions between sets as the homomorphisms so for sets $a$ and $a^{\prime}, \operatorname{Hom}\left(a, a^{\prime}\right)$ is the set of functions $a \rightarrow a^{\prime}$. In the product category Sets $\times$ Sets, the objects are ordered pairs of sets $(a, b)$ and homomorphism $(a, b) \rightarrow\left(a^{\prime}, b^{\prime}\right)$ is just a pair of functions $(f, g)$ where $f: a \rightarrow a^{\prime}$ and $g: b \rightarrow b^{\prime}$.

For an example of an adjunction, consider the product functor $\times:$ Sets $\times$ Sets $\rightarrow$ Sets which takes a pair of sets $(a, b)$ to their Cartesian product $a \times b$ (set of ordered pairs of elements from $a$ and $b)$ and takes a homomorphism $(f, g):(a, b) \rightarrow\left(a^{\prime}, b^{\prime}\right)$ to $f \times g: a \times b \rightarrow a^{\prime} \times b^{\prime}$ where for $x \in a$ and $y \in b, f \times g:(x, y) \longmapsto(f(x), g(y))$.

The maps $f: a \rightarrow a^{\prime}$ in Sets go from one set to one set and the maps $(f, g):(a, b) \rightarrow\left(a^{\prime}, b^{\prime}\right)$ in Sets $\times$ Sets go from a pair of sets to a pair of sets. There is also the idea of a cone $[f, g]: c \rightarrow(a, b)$ of maps that is a pair of maps $f: c \rightarrow a$ and $g: c \rightarrow b$ going from one set $c$ (the point of the cone) in Sets to a pair of sets $(a, b)$ (the base of the cone) in Sets $\times$ Sets. Before the notion of a adjunction was defined by Kan [15, the product of sets $a \times b$ was defined by its universal mapping property. The projection maps $\pi_{a}: a \times b \rightarrow a$ and $\pi_{b}: a \times b \rightarrow b$ define a canonical cone $\left[\pi_{a}, \pi_{b}\right]: a \times b \rightarrow(a, b)$ that is universal in the following sense. Given any other cone $[f, g]: c \rightarrow(a, b)$ from any set $c$ to $(a, b)$, there is a unique homomorphism $\langle f, g\rangle: c \rightarrow a \times b$ in Sets such that the two triangles in the following diagram commute.

\footnotetext{
${ }^{4}$ Then the universal for all the non-self-predicative universals would give rise to Russell's Paradox since it could not be self-predicative or non-self-predicative (Russell [33] p. 80]).
} 


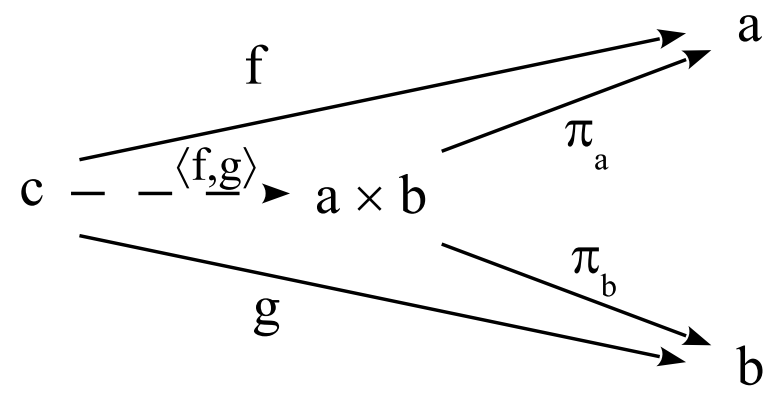

Figure 1: Universal mapping property for the product of sets.

In terms of the self-predicative universals considered in the last section, the property in question is the property of being a cone $[f, g]: c \rightarrow(a, b)$ to $(a, b)$ from any set $c$. The canonical cone of projections $\left[\pi_{a}, \pi_{b}\right]: a \times b \rightarrow(a, b)$ is the self-predicative universal for that property. The participation relation $[f, g] \mu\left[\pi_{a}, \pi_{b}\right]$ is defined as "uniquely factoring through" (as in Figure 1 ). The universal mapping property of the product can then be restated as the universality condition: For any cone $[f, g]$ from any set to a pair of sets,

\section{$[f, g] \mu\left[\pi_{a}, \pi_{b}\right]$ if and only if $[f, g]$ is a cone to $(a, b)$.}

UMP of $a \times b$ stated as a universality condition.

The Hom-set definition of the adjunction for the product functor uses the auxiliary device of a diagonal functor to avoid mentioning the cones and to restrict attention only to the Hom-sets of the two categories. The diagonal functor $\Delta:$ Sets $\rightarrow$ Sets $\times$ Sets in the opposite direction of the product functor just doubles everything so $\Delta(c)=(c, c)$ and $\Delta(f)=(f, f)$. Then the product functor is said to be the right adjoint of the diagonal functor, the diagonal functor is said to be the left adjoint of the product functor, and the two functors together form an adjunction if there is a natural isomorphism between the Hom-sets as follows:

$\operatorname{Hom}_{\text {Sets } \times \text { Sets }}(\Delta(c),(a, b)) \cong \operatorname{Hom}_{\text {Sets }}(c, a \times b)$.

Hom-set definition of the adjunction between the product and diagonal functors.

The diagonal functor $\Delta:$ Sets $\rightarrow$ Sets $\times$ Sets also has a (rather trivial) UMP that can be stated in terms of cones $c \rightarrow(a, b)$ except now we fix $c$ and let $(a, b)$ vary. There is the canoncial cone $\left[1_{c}, 1_{c}\right]: c \rightarrow(c, c)$ and it is universal in the following sense. For any cone $[f, g]: c \rightarrow(a, b)$ from the given $c$ to any pair of sets $(a, b)$, there is a unique homomorphism in Sets $\times$ Sets, namely $(f, g):(c, c) \rightarrow(a, b)$ that factors through the canonical cone $c \rightarrow(c, c)$.

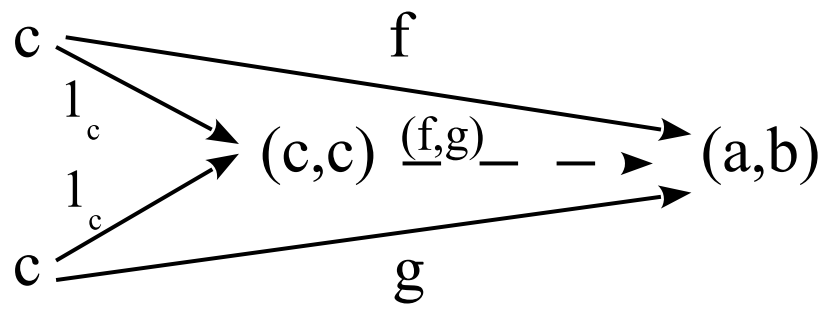

Figure 2: Universal mapping property for diagonal functor.

This product-diagonal adjunction illustrates the general Hom-set definition. Given functors $F$ : $\mathbb{X} \rightarrow \mathbb{A}$ and $G: \mathbb{A} \rightarrow \mathbb{X}$ going each way between categories $\mathbb{X}$ and $\mathbb{A}$, they form an adjunction if there is a natural isomorphism (for objects $X \in \mathbb{X}$ and $A \in \mathbb{A}$ ): 
$\operatorname{Hom}_{\mathbb{A}}(F(X), A) \cong \operatorname{Hom}_{\mathbb{X}}(X, G(A))$

Hom-set definition of an adjunction.

To further analyze adjoints, we need the notion of a "heteromorphism."

\section{$5 \quad$ Heteromorphisms and adjunctions}

We have seen that there are two UMPs (often one is trivial like $\Delta(c)$ in the above example) involved in an adjunction and that the object-to-object maps were always within one category, e.g., in the "Hom-sets" of one category or the other. Using object-to-object maps between objects of different categories (properly called "heteromorphisms" or "chimera morphisms"), the notion of an adjunction can be factored into two representations (or "half-adjunctions" in Ellerman [7, p. 158]), each of which expresses a universal mapping property.

We have already seen one standard example of a heteromorphism or het, namely a cone $[f, g]$ : $c \rightarrow(a, b)$ that goes from an object in Sets to an object in Sets $\times$ Sets. The hets are contrasted with the homs or homomorphisms between objects in the same category. To keep them separate in our notation, we will henceforth use single arrows $\longrightarrow$ for hets and double arrows $\Rightarrow$ for homs 5 Then the UMP for the product functor can be represented as follows.

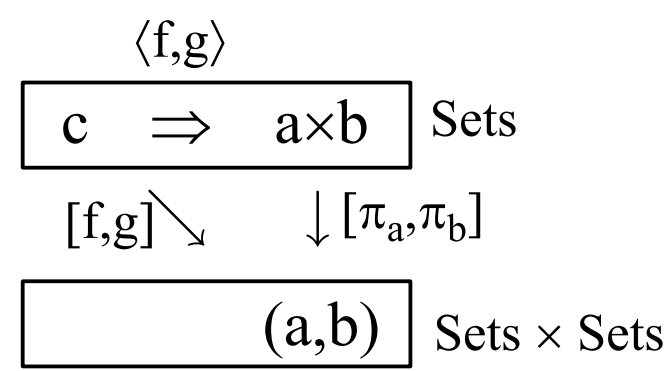

Figure 3: UMP for the product functor

It should be particularly noted that this het-formulation of the UMP for the product does not involve the diagonal functor. If we associate with each $c \in$ Sets and each $(a, b) \in \operatorname{Set} \times$ Sets, the set Het $(c,(a, b))$ of cones or hets $[f, g]: c \rightarrow(a, b)$ then this defines a Het-bifunctor in the same manner as the usual Hom-bifunctor $\operatorname{Hom}_{\text {Sets }}\left(a, a^{\prime}\right)$ or $\operatorname{Hom}_{\text {Sets } \times \text { Sets }}\left((a, b),\left(a^{\prime}, b^{\prime}\right)\right)$ [see the appendix for more details]. Then the UMP for the product functor gives a natural isomorphism based on the pairing: $[f, g] \mapsto\langle f, g\rangle$, so that the Sets-valued functor Het $(c,(a, b))$ is said to be represented on the right by the Sets-valued $\operatorname{Hom}_{\text {Sets }}(c, a \times b)$ :

$$
\operatorname{Het}(c,(a, b)) \cong \operatorname{Hom}_{\text {Sets }}(c, a \times b)
$$

Right representation of the hets $c \rightarrow(a, b)$ by the homs $c \Rightarrow a \times b$.

The trivial UMP for the diagonal functor can also be stated in terms of the cone-hets without reference to the product functor.

\footnotetext{
${ }^{5}$ The hets between objects of different categories are represented as single arrows $(\rightarrow)$ while the homomorphisms or homs between objects in the same category are represented by double arrows $(\Rightarrow)$. The functors between whole categories are also represented by single arrows $(\rightarrow)$. One must be careful not to confuse a functor $F: \mathbb{X} \rightarrow \mathbb{A}$ from a category $\mathbb{X}$ to a category $\mathbb{A}$ with its action on an object $X \in \mathbb{X}$ which would be symbolized $X \longmapsto F(X)$. Moreover since a functor often has a canonical definition, there may well be a canonical het $X \rightarrow F(X)$ or $X \leftarrow F(X)$ but such hets are no part of the definition of the functor itself.
} 


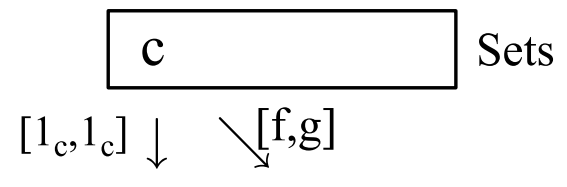

\section{$\Delta(\mathrm{c}) \Rightarrow(\mathrm{a}, \mathrm{b})$ Sets $\times$ Sets \\ $(\mathrm{f}, \mathrm{g})$}

Figure 4: UMP for the diagonal functor

This UMP for the diagonal functor gives a natural isomorphism based on the pairing $(f, g) \longmapsto[f, g]$, so the Sets-valued functor Het $(c,(a, b))$ is said to be represented on the left by the Sets-valued $\operatorname{Hom}_{\text {Sets } \times \text { Sets }}((c, c),(a, b))$ :

$$
\operatorname{Hom}_{\text {Sets } \times \text { Sets }}((c, c),(a, b)) \cong \operatorname{Het}(c,(a, b))
$$

Left representation of the hets $c \rightarrow(a, b)$ by the homs $(c, c) \Rightarrow(a, b)$.

Then the right and left representations of the hets Het $(c,(a, b))$ can be combined to obtain as a consequence the Hom-set definition of the adjunction between the product and diagonal functors:

$\operatorname{Hom}_{\text {Sets } \times \text { Sets }}((c, c),(a, b)) \cong \operatorname{Het}(c,(a, b)) \cong \operatorname{Hom}_{\text {Sets }}(c, a \times b)$
Heteromorphic presentation of the product-diagonal adjunction.

In the general case of adjoint functors $F: \mathbb{X} \rightleftarrows \mathbb{A}: G$, the hets $\operatorname{Het}(X, A)$ from objects $X \in \mathbb{X}$ to objects $A \in \mathbb{A}$ have left and right representations:

$$
\begin{aligned}
& \operatorname{Hom}_{\mathbb{A}}(F(X), A) \cong \operatorname{Het}(X, A) \cong \operatorname{Hom}_{\mathbb{X}}(X, G(A)) \\
& \text { Heteromorphic presentation of a general adjunction. }
\end{aligned}
$$

This is the heterodox treatment of an adjunction first published by Pareigis [28, pp. 60-1] and later rediscovered and developed by the author ([7, p. 130] and [8]). It is "heterodox" since the morphisms between the objects of different categories are not "officially" recognized in the standard presentations of category theory (e.g., 24] or [1]) even though such hets are a common part of mathematical practice (see the appendix for further discussion). Hence the standard Hom-set definition of an adjunction just deletes the Het-middle-term $\operatorname{Het}(X, A)$ to obtain just the het-free or homs-only presentation of an adjunction.

The important advance of the heteromorphic treatment of an adjunction is that the adjunction can be parsed or factored into two parts, the left and right representations, each of which only involves one of the Hom-functors.
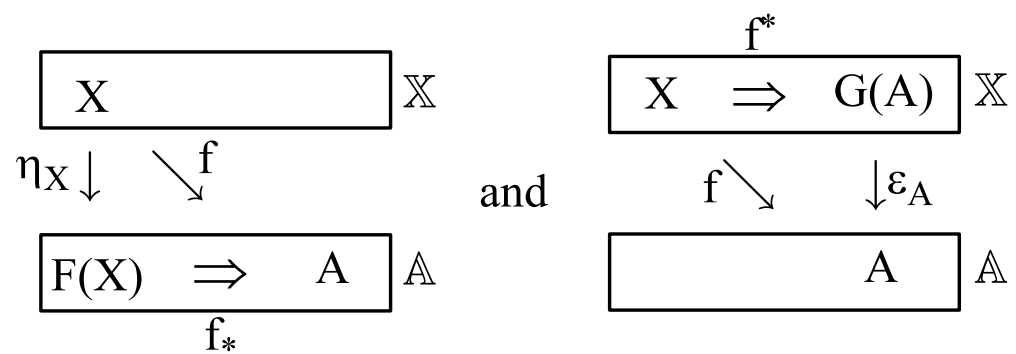

Figure 5: Left and right representations each involving on one of the adjoints $F$ and $G$.

Moreover, the diagrams for the two representations can be glued together at the diagonal het $\searrow^{f}$ into one diagram to give the simple adjunctive square diagram for an adjunction. 


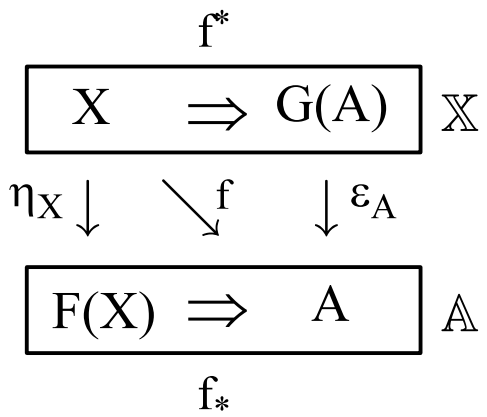

Figure 6: Adjunctive square diagram for the het-treatment of an adjunction.

Every adjunction can be represented (up to isomorphism) in this manner [7, p. 147] so the molecule of an adjunction can be split into two atoms, each of which is a (left or right) representation of a Het-functor. This means that the importance and ubiquity of adjunctions (emphasized above) also passes to the atoms, left or right representations, that make up those molecules. Moreover, it should be noted that each left or right representation defines a self-predicative universal as indicated in the previous example of the het-cones $c \rightarrow(a, b)$.

The main point of this paper is that those atoms, the left and right representations can be recombined in a new way to define a "recombinant construction" cognate to an adjunction, and that is the concept of a "brain functor."

\section{Brain functors}

In many adjunctions, the important fact is expressed by either the left or right representation (e.g., the UMP for the product functor or for the free-group functor considered in the Appendix), with no need for the "auxiliary device" (such as a diagonal or forgetful functor) of the other representation used to express the adjunction in a het-free manner.

Another payoff from analyzing the important but molecular concept of an adjunction into two atomic representations is that we can then reassemble those atomic parts in a new way to define the cognate concept speculatively named a "brain functor."

The basic intuition is to think of one category $\mathbb{X}$ in a representation as the "environment" and the other category $\mathbb{A}$ as an "organism." Instead of representations within each category of the hets going one way between the categories (as in an adjunction), suppose the hets going both ways were represented within one of the categories (the "organism").

Intuitively, a het from the environment to the organism is say, a visual or auditory stimulus. Then a left representation would play the role of the brain in providing the re-cognition or perception (expressed by the intentionality-of-perception slogan: "seeing is seeing-as") of the stimulus as a perception of, say, a tree where the internal re-cognition is represented by the homomorphism $\Rightarrow$ inside the "organism" category. 


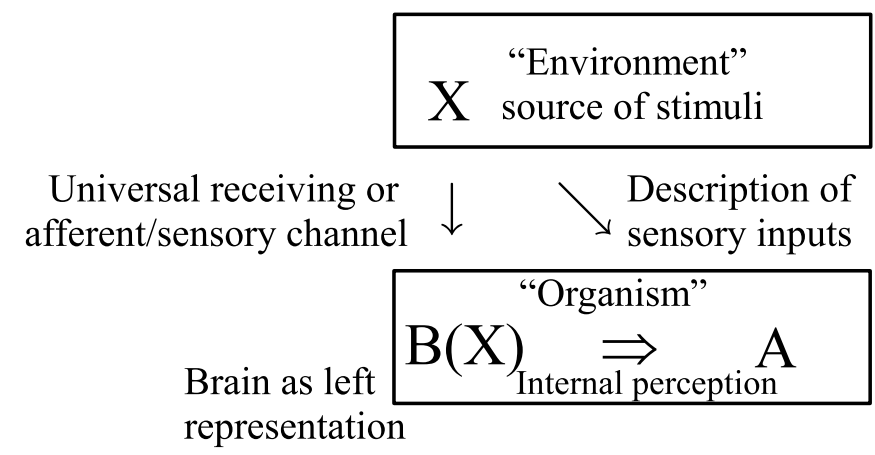

Figure 7: Perceiving brain presented as a left representation.

Perhaps not surprisingly, this mathematically models the old philosophical theme in the Platonic tradition that external stimuli do not give knowledge; the stimuli only trigger the internal perception, recognition, or recollection (as in Plato's Meno) that is knowledge. In De Magistro (The Teacher), the neo-Platonic Christian philosopher Augustine of Hippo developed an argument (in the form of a dialogue with his son Adeodatus) that as teachers teach, it is only the student's internal appropriation of what is taught that gives understanding.

Then those who are called pupils consider within themselves whether what has been explained has been said truly; looking of course to that interior truth, according to the measure of which each is able. Thus they learn,.... But men are mistaken, so that they call those teachers who are not, merely because for the most part there is no delay between the time of speaking and the time of cognition. And since after the speaker has reminded them, the pupils quickly learn within, they think that they have been taught outwardly by him who prompts them. (Augustine, De Magistro, Chapter XIV)

The basic point is the active role of the mind in generating understanding (represented by the internal hom). This is clear even at the simple level of understanding spoken words. We hear the auditory sense data of words in a completely strange language as well as the words in our native language. But the strange words bounce off our minds, like @ $\# \$ \%^{\wedge}$, with no resultant understanding while the words in a familiar language prompt an internal process of generating a meaning so that we understand the words. Thus it could be said that "understanding a language" means there is a left representation for the heard statements in that language, but there is no such internal re-cognition mechanism for the heard auditory inputs in a strange language.

Dually, there are also hets going the other way from the "organism" to the "environment" and there is a similar distinction between mere behavior (e.g., a reflex) and an action that expresses an intention. Mathematically that is described by dualizing or turning the arrows around which gives an acting brain presented as a right representation.

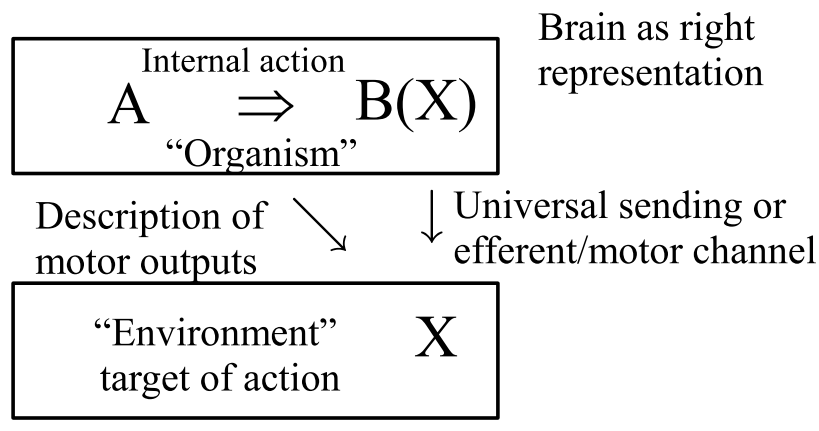


Figure 8: Acting brain as a right representation.

In the heteromorphic treatment of adjunctions, an adjunction arises when the hets from one category $\mathbb{X}$ to another category $\mathbb{A}, \operatorname{Het}(X, A)$ for $X \in \mathbb{X}$ and $A \in \mathbb{A}$, have a right representation, $\operatorname{Het}(X, A) \cong \operatorname{Hom}_{\mathbb{X}}(X, G(A))$, and a left representation, $\operatorname{Hom}_{\mathbb{A}}(F(X), A) \cong \operatorname{Het}(X, A)$. But instead of taking the same set of hets as being represented by two different functors on the right and left, suppose we consider a single functor $B(X)$ that represents the hets $\operatorname{Het}(X, A)$ on the left:

$$
\operatorname{Het}(X, A) \cong \operatorname{Hom}_{\mathbb{A}}(B(X), A),
$$

and represents the hets $\operatorname{Het}(A, X)$ [going in the opposite direction] on the right:

$$
\operatorname{Hom}_{\mathbb{A}}(A, B(X)) \cong \operatorname{Het}(A, X) .
$$

If the hets each way between two categories are represented by the same functor $B(X)$ as left and right representations, then that functor is said to be a brain functor. Thus instead of a pair of functors being adjoint, we have a single functor $B(X)$ with values within one of the categories (the "organism") as representing the two-way interactions, "perception" and "action," between that category and another one (the "environment"). The use of the adjective "brain" is quite deliberate (as opposed to say "mind") since the universal hets going each way between the "organism" and "environment" are part of the definition of left and right representations. In particular, it should be noted how the "turn-around-the-arrows" category-theoretic duality provides a mathematical model for the type of "duality" between:

- sensory or afferent systems (brain furnishing the left representation of the environment to organism heteromorphisms), and

- motor or efferent systems (brain furnishing the right representation of the organism to environment heteromorphisms).

In view of this application, those two universal hets, representing the afferent and efferent nervous systems, might be denoted $\alpha_{X}$ and $\varepsilon_{X}$ as in the following diagrams for the two representations.
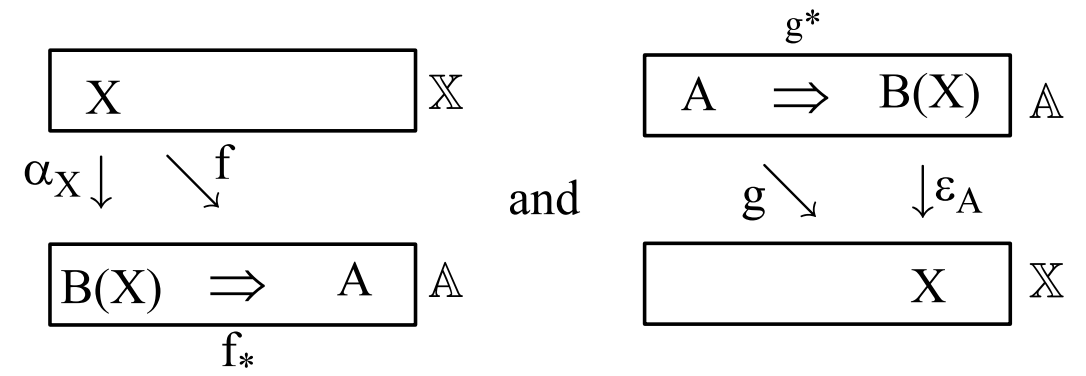

Figure 9: Left and right representation diagrams for the brain functor $B: \mathbb{X} \rightarrow \mathbb{A}$.

We have seen how the adjunctive square diagram for an adjunction can be obtained by gluing together the left and right representation diagrams at the common diagonal $\searrow^{f}$. The diagram for a brain functor is obtained by gluing together the diagrams for the left and right representations at the common values of the brain functor $B(X)$. If we think of the diagram for a representation as right triangle, then the adjunctive square diagram is obtained by gluing two triangles together on the hypotenuses, and the diagram for the brain functor is obtained by gluing two triangles together at the right angle vertices to form the butterfly diagram. 


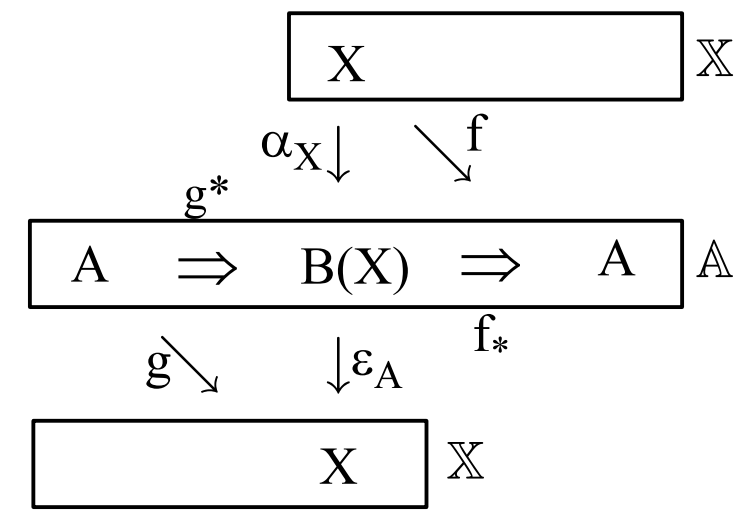

Figure 10: Butterfly diagram combining two representations at the common $B(X)$

If both the triangular "wings" could be filled-out as adjunctive squares, then the brain functor would have left and right adjoints. Thus all functors with both left and right adjoints are brain functors (although not vice-versa). The previous example of the diagonal functor $\Delta:$ Sets $\rightarrow$ Sets $\times$ Sets is a brain functor since the product functor $\times(a, b)=a \times b$ is the right adjoint, and the coproduct or disjoint union functor $\biguplus(a, b)=a \biguplus b$ is the left adjoint. The underlying set functor (see Appendix) that takes a group $G$ to its underlying set $U(G)$ is a rather trivial example of a brain functor that does not arise from having both a left and right adjoint. It has a left adjoint (the free group functor) so $U$ provides a right representation for the set-to-group maps or hets $X \rightarrow G$. Also it trivially provides a left representation for the hets $G \rightarrow X$ but has no right adjoint.

In the butterfly diagram below, we have labelled the diagram for the brain as the language faculty for understanding and producing speech.

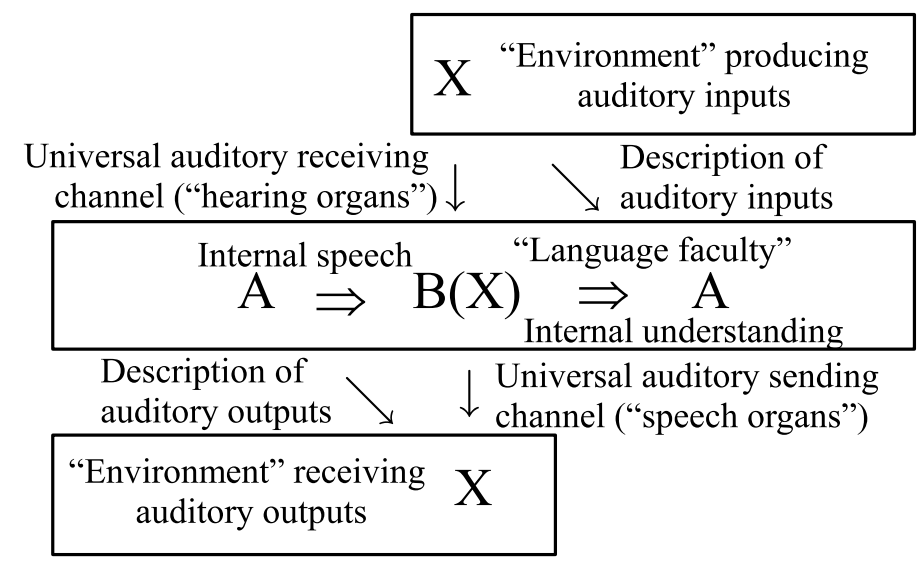

Figure 11: Brain functor butterfly diagram interpreted as language faculty.

Wilhelm von Humboldt recognized the symmetry between the speaker and listener, which in the same person is abstractly represented as the dual functions of the "selfsame power" of the language faculty in the above butterfly diagram.

Nothing can be present in the mind (Seele) that has not originated from one's own activity. Moreover understanding and speaking are but different effects of the selfsame power of speech. Speaking is never comparable to the transmission of mere matter (Stoff). In the person comprehending as well as in the speaker, the subject matter must be developed by the individual's own innate power. What the listener receives is merely the harmonious vocal stimulus.[11, p. 102] 


\section{A mathematical example of a brain functor}

A non-trivial mathematical example of a brain functor is provided by the functor taking a finite set of vector spaces $\left\{V_{i}\right\}_{i=1, \ldots, n}$ over the same field (or $R$-modules over a ring $R$ ) to the product $\prod_{i} V_{i}$ of the vector spaces. Such a product is also the coproduct $\sum_{i} V_{i}[12$, p. 173] and that space may be written as the biproduct:

$$
V_{1} \oplus \ldots \oplus V_{n} \cong \prod_{i} V_{i} \cong \sum_{i} V_{i} .
$$

The het from a set of spaces $\left\{V_{i}\right\}$ to a single space $V$ is a cocone of vector space maps $\left\{V_{i} \Rightarrow V\right\}$ and the canonical such het is the set of canonical injections $\left\{V_{i} \Rightarrow V_{1} \oplus \ldots \oplus V_{n}\right\}$ (taking the "brain" as a coproduct) with the "brain" at the point of the cocone. The perception left representation then might be taken as conceptually representing the function of the brain as integrating multiple sensory inputs into an interpreted perception 6

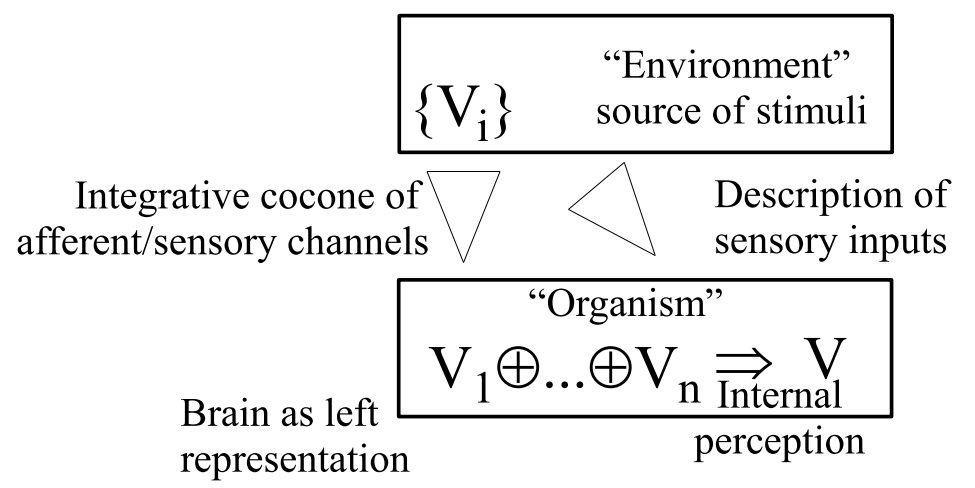

Figure 12: Brain as integrating sensory inputs into a perception.

Dually, a het from single space $V$ to a set of vector spaces $\left\{V_{i}\right\}$ is a cone $\left\{V \Rightarrow V_{i}\right\}$ with the single space $V$ at the point of the cone, and the canonical het is the set of canonical projections (taking the "brain" as a product) with the "brain" as the point of the cone: $\left\{V_{1} \oplus \ldots \oplus V_{n} \Rightarrow V i\right\}$. The action right representation then might be taken as conceptually representing the function of the brain as integrating or coordinating multiple motor outputs in the performance of an action.

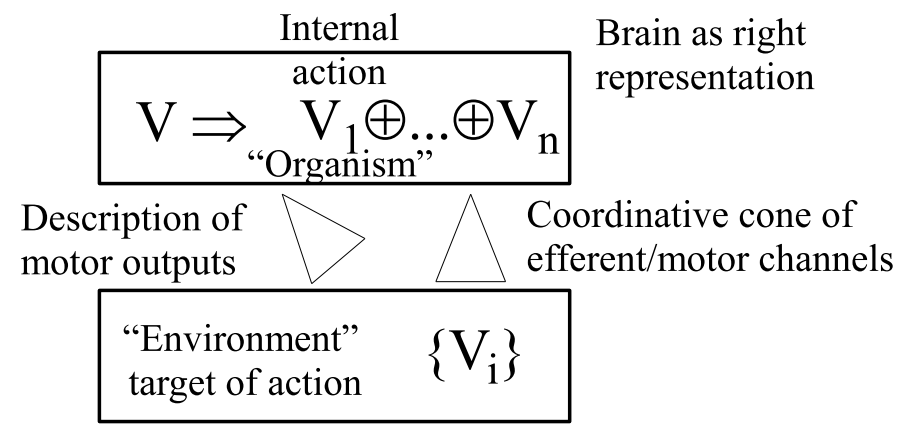

Figure 13: Brain as coordinating motor outputs into an action.

Putting the two representations together gives the butterfly diagram for a brain.

\footnotetext{
${ }^{6}$ The cocones and cones are represented in the diagrams using cone shapes.
} 


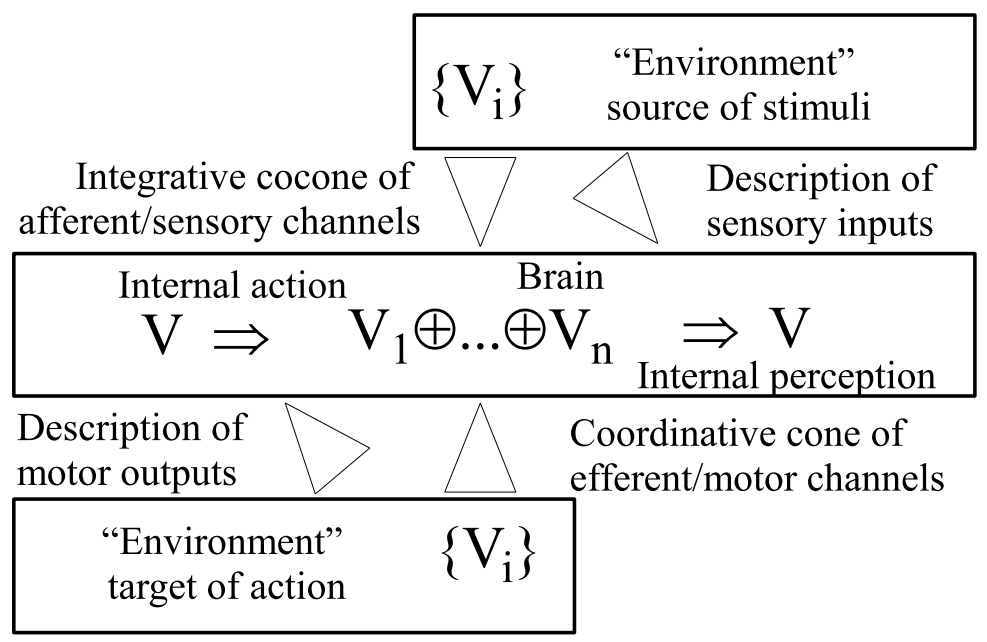

Figure 14: Conceptual model of a perceiving and acting brain.

This gives a conceptual model of a single organ that integrates sensory inputs into a perception and coordinates motor outputs into an action, i.e., a brain.

\section{Conclusion}

In view of the success of category theory in modern mathematics, it is perfectly natural to try to apply it in the life and cognitive sciences. Many different approaches need to be tried to see which ones, if any, will find "where theory lives" (and will be something more than just applying biological names to bits of pure math). The approach developed here differs from other approaches in several ways, but the most basic difference is the use of heteromorphisms to represent interactions between quite different entities (i.e., objects in different categories). Heteromorphisms also provide the natural setting to formulate universal mapping problems and their solutions as left or right representations of hets. In spite of abounding in the wilds of mathematical practice, hets are not recognized in the orthodox presentations of category theory. One consequence is that the notion of an adjunction appears as one atomic concept that cannot be factored into separate parts. But that is only a artifact of the homs-only treatment. The heteromorphic treatment shows that an adjunction factors naturally into a left and right representation of the hets going from one category to another-where, in general, one representation might exist without the other. One benefit of this heteromorphic factorization is that the two atomic concepts of left and right representations can then be recombined in a new way to form the cognate recombinant concept of a brain functor. The main conclusion of the paper is that this concept of a brain functor seems to fit very well as an abstract and conceptual but non-trivial description of the dual universal functions of a brain, perception (using the sensory or afferent systems) and action (using the motor or efferent systems).

\section{Mathematical Appendix: Are hets really necessary in cat- egory theory?}

Since the concept of a brain functor requires hets for its formulation, it is important to consider the role of hets in category theory. The homomorphisms or homs between the objects of a category $\mathbb{X}$ are given by a hom bifunctor $H_{\mathbb{X}}: \mathbb{X}^{o p} \times \mathbb{X} \rightarrow$ Sets. In the same manner, the heteromorphisms 
or hets from the objects of a category $\mathbb{X}$ to the objects of a category $\mathbb{A}$ are given by a het bifunctor Het $: \mathbb{X}^{o p} \times \mathbb{A} \rightarrow$ Sets 7

The Het-bifunctor gives the rigorous way to handle the composition of a het $f: x \rightarrow a$ in Het $(x, a)$ [thin arrows $\rightarrow$ for hets] with a homomorphism or hom $g: x^{\prime} \Longrightarrow x$ in $X$ [thick Arrows $\Longrightarrow$ for homs] and a hom $h: a \Longrightarrow a^{\prime}$ in $A$. For instance, the composition $x^{\prime} \stackrel{g}{\Longrightarrow} x \stackrel{f}{\rightarrow} a$ is the het that is the image of $f$ under the map: Het $(g, a): \operatorname{Het}(x, a) \rightarrow \operatorname{Het}\left(x^{\prime}, a\right)$. Similarly, the composition $x \stackrel{f}{\rightarrow} a \stackrel{h}{\Longrightarrow} a^{\prime}$ is the het that is the image of $f$ under the map: Het $(x, h): \operatorname{Het}(x, a) \rightarrow \operatorname{Het}\left(x, a^{\prime}\right)$ 8

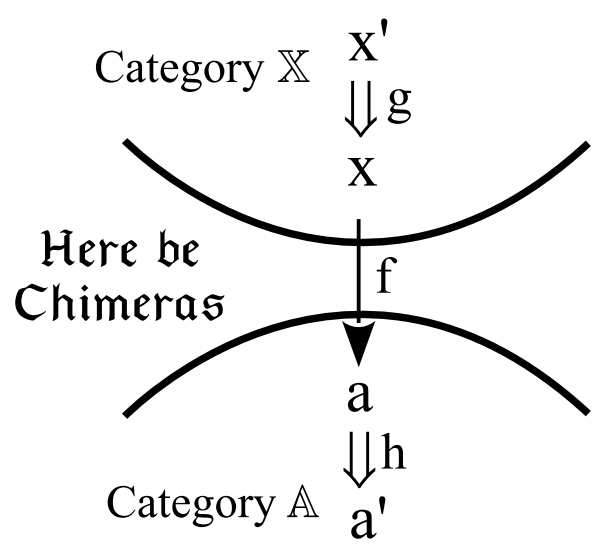

Figure 15: Composition of a het with a hom on either end

This is all perfectly analogous to the use of Hom-functors to define the composition of homs. Since both homs and hets (e.g., injection of generators into a group) are common morphisms used in mathematical practice, both types of bifunctors formalize standard mathematical machinery.

\subsection{Chimeras in the wilds of mathematical practice}

The homs-only orientation may go back to the original conception of category theory "as a continuation of the Klein Erlanger Programm, in the sense that a geometrical space with its group of transformations is generalized to a category with its algebra of mappings." [5, p. 237] While chimeras do not appear in the orthodox "ontological zoo" of category theory, they abound in the wilds of mathematical practice. In spite of the reference to "Working Mathematician" in the title of Mac Lane's text 24, one might seriously doubt that any working mathematician would give, say, the universal mapping property of free groups using the "device" of the underlying set functor $U$ instead of the traditional description given in the left representation diagram (which does not even mention $U$ ) as can be seen in most any non-category-theoretic text that treats free groups. For instance, consider the following description in Nathan Jacobson's text [13, p. 69].

To summarize: given the set $X=\left\{x_{1}, \ldots, x_{r}\right\}$ we have obtained a map $x_{i} \rightarrow \bar{x}_{i}$ of $X$ into a group $F G^{(r)}$ such that if $G$ is any group and $x_{i} \rightarrow a_{i}, 1 \leq i \leq r$ is any map of $X$ into $G$ then we have a unique homomorphism of $F G^{(r)}$ into $G$, making the following diagram commutative:

\footnotetext{
${ }^{7}$ Although often with a somewhat different interpretation, the Sets-valued profunctors [16], distributors [2], or correspondences [22] p. 96] are formally the same as het bifunctors.

${ }^{8}$ The definition of a bifunctor also insures the associativity of composition so that schematically: hom $\circ($ het $\circ$ hom $)=$ (hom o het) o hom.
} 


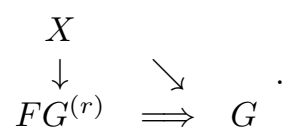

In Jacobson's diagram, only the $F G^{(r)} \Longrightarrow G$ morphism is a group homomorphism; the vertical and diagonal arrows are called "maps" and are set-to-group hets so it is the diagram for a left representation 9

\subsection{Hets as "homs" in a collage category}

The notion of a homomorphism is so general that hets can always be recast as "homs" in a larger category variously called a directly connected category [28, p. 58] (since Pareigis calls the het bifunctor a "connection"), a cograph category [35, or, more colloquially, a collage category (since it combines quite different types of objects and morphisms into one category in total disregard of any connection to the Erlangen Program). The collage category of a het bifunctor Het : $\mathbb{X}^{o p} \times \mathbb{A} \rightarrow$ Sets, denoted $\mathbb{X} \star^{\text {Het }} \mathbb{A}[22$, p. 96], has as objects the disjoint union of the objects of $\mathbb{X}$ and $\mathbb{A}$. The homs of the collage category are defined differently according to the two types of objects. For $x$ and $x^{\prime}$ objects in $\mathbb{X}$, the homs $x \Rightarrow x^{\prime}$ are the elements of $\operatorname{Hom}_{\mathbb{X}}\left(x, x^{\prime}\right)$, the hom bifunctor for $\mathbb{X}$, and similarly for objects $a$ and $a^{\prime}$ in $\mathbb{A}$, the homs $a \Rightarrow a^{\prime}$ are the elements of $\operatorname{Hom}_{\mathbb{A}}\left(a, a^{\prime}\right)$. For the different types of objects such as $x$ from $\mathbb{X}$ and $a$ from $\mathbb{A}$, the "homs" $x \Rightarrow a$ are the elements of Het $(x, a)$ and there are no homs $a \Rightarrow x$ in the other direction in the collage category.

Does the collage category construction show that "hets" are unnecessary in category theory and that homs suffice? Since all the information given in the het bifunctor has been repackaged in the

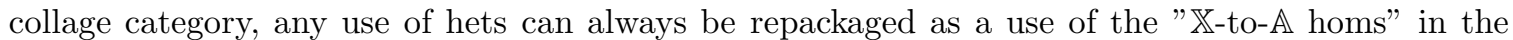
collage category $\mathbb{X} \star^{\text {Het }} \mathbb{A}$. In any application, like the previous example of the universal mapping property (UMP) of the free-group functor as a left representation, one must distinguish between the two types of objects and the three types of "homs" in the collage category.

Suppose in Jacobson's example, one wanted to "avoid" having the different "maps" and group homomorphisms by formulating the left representation in the collage category formed from the category of Sets, the category of groups Grps, and the het bifunctor, Het : Sets ${ }^{o p} \times$ Grps $\rightarrow$ Sets, for set-to-group maps. Since the UMP does not hold for arbitrary objects and homs in the collage category, Sets $\star^{\text {Het }}$ Grps, one would have to differentiate between the "set-type objects" $X$ and the "group-type objects" $G$ as well as between the "mixed-type homs" in $\operatorname{Hom}(X, G)$ and the "puretype homs" in Hom $\left(F G^{(r)}, G\right)$. Then the left representation UMP of the free-group functor could be formulated in the het-free collage category Sets $\star^{\text {Het }}$ Grps as follows.

For every set-type object $X$, there is a group-type object $F(X)$ and a mixed-type hom $\eta_{X}: X \Rightarrow F(X)$ such that for any mixed-type hom $f: X \Rightarrow G$ from the set-type object $X$ to any group-type object $G$, there is a unique pure-type hom $f_{*}: F(X) \Rightarrow G$ such that $f=f_{*} \eta_{X}$.

Thus the answer to the question "Are hets really necessary?" is "No!"-since one can always use sufficient circumlocutions with the different types of "homs" in a collage category. Jokes aside, the collage category formulation is essentially only a reformulation of the left representation UMP using clumsy circumlocutions. Working mathematicians use phrases like "mappings" or "morphisms" to refer to hets in contrast to homomorphisms-and "mixed-type homs" does not seem to be improved phraseology for hets.

There is, however, a more substantive point, i.e., the general UMPs of left or right representations show that the hets between objects of different categories can be represented by homs within the codomain category or within the domain category, respectively. If one conflates the hets and homs

\footnotetext{
${ }^{9}$ We modified Jacobson's diagram according to our het-hom convention for the arrows. Similar examples of hets can be found in the Mac Lane-Birkhoff's text [25].
} 
in a collage category, then the point of the representation is rather obscured (since it is then one set of "homs" in a collage category being represented by another set of homs in the same category).

\subsection{What about the homs-only UMPs in adjunctions?}

There is another het-avoidance device afoot in the homs-only treatment of adjunctions. For instance, the left-representation UMP of the free-group functor can, for each $X \in$ Sets, be formulated as the natural isomorphism: $\operatorname{Hom}_{\text {Grps }}(F(X), G) \cong \operatorname{Het}(X, G)$. But if we fix $G$ and use the underlying set functor $U:$ Grps $\rightarrow$ Sets, then there is trivially the right representation: $\operatorname{Het}(X, G) \cong \operatorname{Hom}_{\text {Sets }}(X, U(G))$. Putting the two representations together, we have the heteromorphic treatment of an adjunction first formulated by Pareigis [28]:

$$
\operatorname{Hom}_{G r p s}(F(X), G) \cong \operatorname{Het}(X, G) \cong \operatorname{Hom}_{\text {Sets }}(X, U(G)) .
$$

If we delete the het middle term, then we have the usual homs-only formulation of the free-group adjunction,

$$
\operatorname{Hom}_{\text {Grps }}(F(X), G) \cong \operatorname{Hom}_{\text {Sets }}(X, U(G)),
$$

without any mention of hets. Moreover, the het-avoidance device of the underlying set functor $U$ allows the UMP of the free group functor to be reformulated with sufficient circumlocutions to avoid mentioning hets.

For each set $X$, there is a group $F(X)$ and a set hom $\eta_{X}: X \Rightarrow U(F(X))$ such that for any set hom $f: X \Rightarrow U(G)$ from the set $X$ to the underlying set $U(G)$ of any group $G$, there is a unique group hom $f_{*}: F(X) \Rightarrow G$ over in the other category such that the set hom image $U\left(f_{*}\right)$ of the group hom $f_{*}$ back in the original category satisfies $f=U\left(f_{*}\right) \eta_{X} 10$

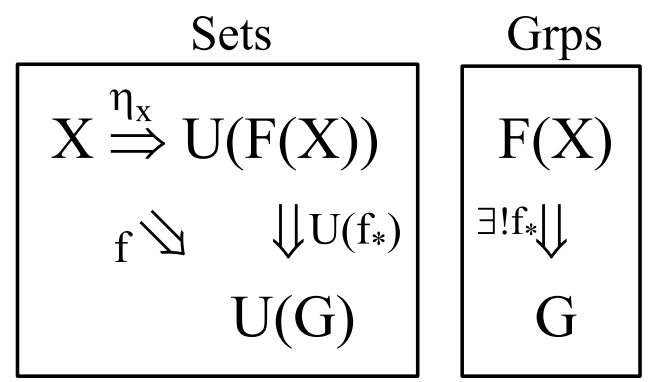

Figure 16: Over-and-back diagram for free group adjunction

Such het-avoidance circumlocutions have no structural significance since there is a general adjunction representation theorem [7, p. 147] that all adjoints can be represented, up to isomorphism, as arising from the left and right representations of a het bifunctor.

\subsection{Are all UMPs part of adjunctions?}

Even though the homs-only formulation of an adjunction only ignores the underlying hets (due to the adjunction representation theorem), is that formulation sufficient to give all UMPs? Or are there important universal constructions that are not either left or right adjoints?

\footnotetext{
${ }^{10}$ Even the "over-and-back" formulation using two different categories could be avoided by using the further circumlocutions of the only pure-type homs in the single collage category.
} 
Probably the most important example is the tensor product. The universal mapping property of the tensor product is particularly interesting since it is a case where the heteromorphic treatment of the UMP is forced (under one disguise or another). The tensor product functor $\otimes:\langle A, B\rangle \longmapsto A \otimes B$ is not a left adjoint so the usual device of using the other functor (e.g., a forgetful or diagonal functor) to avoid mentioning hets is not available.

For $A, B, C$ modules (over some commutative ring $R$ ), one category is the product category $\operatorname{Mod}_{R} \times \operatorname{Mod}_{R}$ where the objects are ordered pairs $\langle A, B\rangle$ of $R$-modules and the other category is just the category $\operatorname{Mod}_{R}$ of $R$-modules. The values of the Het-bifunctor Het $(\langle A, B\rangle, C)$ are the bilinear functions $A \times B \rightarrow C$. Then the tensor product functor $\otimes: \operatorname{Mod}_{R} \times \operatorname{Mod}_{R} \rightarrow \operatorname{Mod}_{R}$ given by $\langle A, B\rangle \longmapsto A \otimes B$ gives a left representation:

$$
\operatorname{Hom}_{M o d}(A \otimes B, C) \cong \operatorname{Het}(\langle A, B\rangle, C)
$$

that characterizes the tensor product. The canonical het $\eta_{\langle A, B\rangle}: A \times B \rightarrow A \otimes B$ is the image under the left-representation isomorphism of the identity hom $1_{A \otimes B}$ obtained by taking $C=A \otimes B$, so we have:

$$
\begin{array}{ccc}
\langle A, B\rangle & & \\
\eta_{\langle A, B\rangle} \downarrow & \searrow^{f} & \\
A \otimes B & \rightleftharpoons & \Longrightarrow f_{*}
\end{array}
$$

Left representation diagram to characterize tensor products

where the single arrows are the bilinear hets and the thick Arrow is a module homomorphism within the category $\operatorname{Mod}_{R}$.

For instance, in Mac Lane and Birkhoff's Algebra textbook [25, they explicitly use hets (bilinear functions) starting with the special case of an $R$-module $A$ (for a commutative ring $R$ ) and then stating the universal mapping property of the tensor product $A \otimes R \cong A$ using the left representation diagram [25, p. 318]-like any other working mathematicians. For any $R$-module $A$, there is an $R$ module $A \otimes R$ and a canonical bilinear het $h_{0}: A \times R \rightarrow A \otimes R$ such that given any bilinear het $h: A \times R \rightarrow C$ to an $R$-module $C$, there is a unique $R$-module hom $t: A \otimes R \Longrightarrow C$ such that the following diagram commutes.

$$
\begin{array}{lll}
A \times R & & \\
h_{0} \downarrow & \searrow \\
A \otimes R & \stackrel{\Xi ! t}{ } &
\end{array}
$$

Left representation diagram of special case of tensor product.

\section{References}

[1] Awodey, Steve. 2006. Category Theory. Oxford: Clarendon Press.

[2] Bénabou, J. 1973. Les distributeurs. Report 33. Université Catholique de Louvain: Institut de Mathématique Pure et appliquée.

[3] Boolos, George. 1971. The Iterative Conception of Set. The Journal of Philosophy 68, (April $22): 215-31$.

[4] Ehresmann, A.C., and J.P. Vanbremeersch. 2007. Memory Evolutive Systems: Hierarchy, Emergence, Cognition. Amsterdam: Elsevier.

[5] Eilenberg, Samuel, and Saunders Mac Lane. 1945. General Theory of Natural Equivalences. Transactions of the American Mathematical Society. 58 (2): 231-94. 
[6] Ellerman, David 1988. Category Theory and Concrete Universals. Erkenntnis. 28: 409-29

[7] Ellerman, David. 2006. A Theory of Adjoint Functors-with Some Thoughts on Their Philosophical Significance. In What Is Category Theory?, edited by G. Sica, 127-83. Milan: Polimetrica.

[8] Ellerman, David 2007. Adjoints and Emergence: applications of a new theory of adjoint functors. Axiomathes. 17 (1 March): 19-39.

[9] Goldblatt, Robert 2006 (1984). Topoi: the Categorical Analysis of Logic (revised ed.). Mineola NY: Dover.

[10] Halford, G. S., and W. H. Wilson. 1980. A Category Theory Approach to Cognitive Development. Cognitive Psychology 12 (3): 356-411.

[11] Humboldt, Wilhelm von. 1997 (1836). The Nature and Conformation of Language. In The Hermeneutics Reader, edited by Kurt Mueller-Vollmer, 99-105. New York: Continuum.

[12] Hungerford, Thomas W. 1974. Algebra. New York: Springer-Verlag.

[13] Jacobson, Nathan. 1985. Basic Algebra I. 2nd ed. New York: W.H. Freeman.

[14] Kainen, P.C. 2009. On the Ehresmann-Vanbremeersch Theory and Mathematical Biology. Axiomathes. 19: 225-44.

[15] Kan, Daniel 1958. Adjoint Functors. Transactions of the American Mathematical Society. 87 (2): 294-329.

[16] Kelly, Max. 1982. Basic Concepts of Enriched Category Theory. Cambridge UK: Cambridge University Press.

[17] Lambek, J. 1981. The Influence of Heraclitus on Modern Mathematics. In Scientific Philosophy Today: Essays in Honor of Mario Bunge, edited by J. Agassi and R. S. Cohen, 111-21. Boston: D. Reidel Publishing Co.

[18] Lawvere, F. William 1969. Adjointness in Foundations. Dialectica. 23: 281-95.

[19] Lawvere, F. William, and Stephen Schanuel. 1997. Conceptual Mathematics: A First Introduction to Categories. New York: Cambridge University Press.

[20] Louie, A. H. 1985. Categorical System Theory. In Theoretical Biology and Complexity: Three Essays on the Natural Philosophy of Complex Systems, edited by Robert Rosen, 68-163. Orlando FL: Academic Press.

[21] Louie, A. H., and Roberto Poli. 2011. The Spread of Hierarchical Cycles. International Journal of General Systems. 40 (3 April): 237-61.

[22] Lurie, Jacob. 2009. Higher Topos Theory. Princeton NJ: Princeton University Press.

[23] Mac Lane, Saunders. 1948. Groups, Categories, and Duality. Proc. Nat. Acad. Sci. U.S.A. 34 (6): 263-67.

[24] Mac Lane, Saunders. 1971. Categories for the Working Mathematician. New York: SpringerVerlag.

[25] Mac Lane, Saunders, and Garrett Birkhoff. 1988. Algebra. Third edition. New York: Chelsea.

[26] Magnan, Francois and Gonzalo E. Reyes 1994. Category Theory as a Conceptual Tool in the Study of Cognition. In The Logical Foundations of Cognition. John Macnamara and Gonzalo E. Reyes eds., New York: Oxford University Press: 57-90. 
[27] Makkai, Michael. 1999. Structuralism in Mathematics. In Language, Logic, and Concepts: Essays in Memory of John Macnamara, edited by R. Jackendoff, P. Bloom, and K. Wynn, 43-66. Cambridge: MIT Press (A Bradford Book).

[28] Pareigis, Bodo. 1970. Categories and Functors. New York: Academic Press.

[29] Phillips, Steven. 2014. Analogy, Cognitive Architecture and Universal Construction: A Tale of Two Systematicities. PLOS ONE 9 (2): 1-9.

[30] Phillips, Steven, and William H. Wilson. 2014. Chapter 9: A Category Theory Explanation for Systematicity: Universal Constructions. In Systematicity and Cognitive Architecture, edited by P. Calvo and J. Symons, 227-49. Cambridge, MA: MIT Press.

[31] Rosen, Robert. 1958. The Representation of Biological Systems from the Standpoint of the Theory of Categories. Bulletin of Mathematical Biophysics. 20 (4): 317-42.

[32] Rosen, Robert. 2012. Anticipatory Systems: Philosophical, Mathematical, and Methodological Foundations. Second Ed. New York: Springer.

[33] Russell, Bertrand. 2010 (1903). Principles of Mathematics. London: Routledge Classics.

[34] Samuel, Pierre. 1948. On Universal Mappings and Free Topological Groups. Bull. Am. Math. Soc. 54 (6): 591-98.

[35] Shulman, Mike. 2011. Cograph of a Profunctor. Ncatlab.org. Accessed July 26, 2015. http://ncatlab.org/nlab/show/cograph+of+a+profunctor.

[36] Taylor, Paul 1999. Practical Foundations of Mathematics. Cambridge UK: Cambridge University Press.

[37] Wood, Richard J. 2004. Ordered Sets via Adjunctions. In Categorical Foundations. Encyclopedia of Mathematics and Its Applications Vol. 9\%. Maria Cristina Pedicchio and Walter Tholen eds., Cambridge: Cambridge University Press: 5-47.

[38] Zafiris, E. 2012. Rosen's Modelling Relations via Categorical Adjunctions. International Journal of General Systems 41 (5): 439-74. 